\title{
The structure and associated formation mechanism of deformation twins in Cu-Nb alloy
}

\author{
Ruoshan Lei ${ }^{1, a,{ }^{*}}$, Guangruan Chen ${ }^{1, b}$, Mingpu Wang ${ }^{2, c}$, Shiqing $X u^{1, d}$, \\ Huanping Wang ${ }^{1, \mathrm{e}}$ \\ ${ }^{1}$ College of Materials Science and Engineering, China Jiliang University, Hangzhou, P. R. China \\ ${ }^{2}$ School of Materials Science and Engineering, Central South University, Changsha, P. R. China \\ a leiruo@163.com, ${ }^{\text {b }}$ cgrzdd@163.com, ${ }^{\text {c }}$ wangmp@cus.edu.cn, ${ }^{\mathrm{d}}$ sxucjlu@163.com, \\ e wanghuanping@cjlu.edu.cn
}

Keywords: deformation twin; nanostructure; mechanical alloying; Cu-Nb alloy

\begin{abstract}
The microstructure and associated formation mechanisms of deformation twins (DTs) in $\mathrm{Cu}-\mathrm{Nb}$ alloy prepared by mechanical alloying (MA) at room temperature have been investigated by transmission electron microscope (TEM) and high-resolution TEM (HRTEM) observations. The results show that DTs form in Cu grains with sizes around or below $100 \mathrm{~nm}$. Here, two different types of twins have been observed. One type of twins is nucleated via partial dislocation emission from grain boundaries (GBs). A dislocation based model reveals that decreasing grain size and increasing stress concentration benefit the partial dislocation emission. The other type of twins is formed via the splitting and migration of GBs. These twinning mechanisms are totally different with that accessible to coarse-grained $\mathrm{Cu}$.
\end{abstract}

\section{Introduction}

Deformation twinning seldom occurs in coarse-grain face-centered cubic (fcc) metals with medium-to-high stacking fault energy (SFE), such as $\mathrm{Al}, \mathrm{Ni}$ and $\mathrm{Cu}$, since the slip deformation is dominant [1]. However, recent molecular dynamic (MD) simulations and experiments have revealed that DT becomes easier in $\mathrm{Al}$ and $\mathrm{Cu}$ with grain size of several tens of nanometers [2-4]. Due to the strong strengthening effect of twin boundaries, the nanosized twin structure can increase the strength of the materials without ductility deterioration, which has attracted considerable attentions [5].

$\mathrm{Cu}-\mathrm{Nb}$ alloys prepared by MA and subsequent consolidation are reported to exhibit a good combination of strength and electrical conductivity, which find a wide application in electronic devices [6-9]. In the past, many studies have focused on the solid solubility extension mechanism and the strengthening mechanism in $\mathrm{Cu}-\mathrm{Nb}$ alloy. But, the occurrence of deformation twinning and also its formation mechanisms in $\mathrm{Cu}-\mathrm{Nb}$ alloys have seldom been investigated. In this study, we report TEM and HRTEM observations of the DTs in Cu-Nb alloy processed by MA. The associated formation mechanisms of DT in Cu-Nb alloy have also been investigated.

\section{Experimental methods}

Commercially pure $\mathrm{Cu}$ powders ( $>99.6 \mathrm{wt} . \%$ ) and Nb powders ( $>99.4 \mathrm{wt} . \%$ ) were used and mixed to the desired $\mathrm{Cu}-10 \mathrm{wt} . \% \mathrm{Nb}$ alloy. The MA process was performed in a QM-1SP4 planetary ball mill with stainless steel containers and balls at a milling speed of 300 rpm under an argon atmosphere at room temperature. The powder-to-ball weight ratio was 1:15 in each vial. The MA process was interrupted for $20 \mathrm{~min}$ after every $20 \mathrm{~min}$ of milling to avoid excessive heating. After different milling times, several powders were extracted for microstructural investigations. TEM samples were prepared by mechanical polishing and Ar ion beam milling. TEM and HRTEM images were obtained using a JEOL JEM-2100 with a point resolution of $1.9 \AA$ and at an operating voltage of $200 \mathrm{kV}$. And the HRTEM images have been analyzed using a Gatan Digital Micrograph software (v 3.11.2). 


\section{Results and discussion}

Fig. 1(a) shows the TEM image of the DTs in the sample milled for $30 \mathrm{~h}$. It can be seen that DTs are formed in the grains with size around or below $100 \mathrm{~nm}$ as marked by the white arrows. DTs have also been found in the milled pure $\mathrm{Cu}$ powders with crystalline grain size smaller than $60 \mathrm{~nm}$ [10]. The relatively larger critical size for deformation twinning in $\mathrm{MA}$-ed $\mathrm{Cu}$ - $\mathrm{Nb}$ alloys may be attributed to the effect of Nb solutes. Fig. 1(b) is a HRTEM image of the region marked by the white frame in Fig. 1(a), which shows that a DT nucleated in the Cu grain with size of $154 \mathrm{~nm}$. The fast Fourier transform pattern of Fig. 1(b) demonstrates the twin relationship among the bands and the matrix, and the twin boundary is parallel to the $\{111\}_{\mathrm{Cu}}$ plane. It is obvious that the partial dislocations (labeled as “ ”) nucleated at the GB are propagating into the grain interior, making the further growth of the twin, which indicates that the twin may nucleate at GB and grow via successive partial dislocation emission.
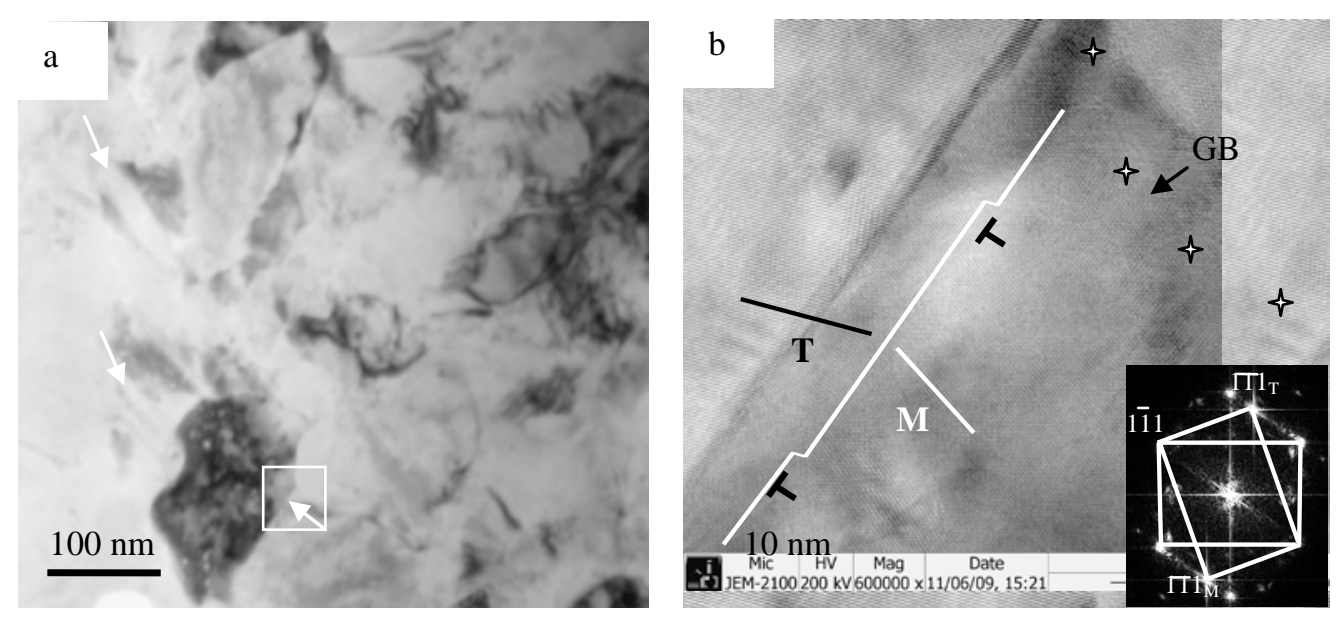

Figure 1. (a) TEM image of DTs in the Cu-Nb powders milled for $30 \mathrm{~h}$; (b) HRTEM image of the region marked by the white box in (a), inset is the Fourier-transformed image confirming the twin relationship.

TEM observations provide direct evidences confirming the occurrence of deformation twinning in MA-ed Cu-Nb alloy with sizes around or below $100 \mathrm{~nm}$ via partial dislocation emission. Huang et al. have proposed a simple model, which compares the shear stress required to initiate Shockley partial dislocation $\left(\tau_{\mathrm{p}}\right)$ with that needed to nucleate a full dislocation $\left(\tau_{\mathrm{s}}\right)$ from GBs [3]. In the model, $\tau_{\mathrm{s}}$ can be determined by:

$$
\tau_{\mathrm{s}}=\frac{2 \alpha \mu \mathrm{b}}{D}
$$

And $\tau_{\mathrm{p}}$ can be calculated by:

$$
\mathrm{n} \tau_{\mathrm{p}}=\frac{2 \alpha \mu \mathrm{b}_{1}}{\mathrm{D}}+\frac{\gamma}{\mathrm{b}_{1}}
$$

where $n$ is a stress concentration factor, $\gamma$ is the SFE, $\mu$ is the shear modulus, $D$ is the crystallite size, $\boldsymbol{b}$ and $\boldsymbol{b}_{1}$ are the Burgers vector of perfect dislocation and Shockley partial dislocation in the $\mathrm{Cu}$ matrix, respectively. The parameter $\alpha$ represents the dislocation character with $\alpha=0.5$ for edge dislocation and $\alpha=1.5$ for screw dislocation, respectively [11]. $\tau_{\mathrm{p}}$ becomes smaller than $\tau_{\mathrm{s}}$, when the grain size decreases to be smaller than the critical value $D_{c}$ determined by equating Eqs. (1) and (2): 


$$
\mathrm{D}_{\mathrm{c}}=\frac{2 \alpha \mu\left(\mathrm{nb}-\mathrm{b}_{1}\right) \mathrm{b}_{1}}{\gamma} \text {. }
$$

To simply the calculation, we ignore the influence of Nb solutes on the SFE of $\mathrm{Cu}$. For pure $\mathrm{Cu}$, $\gamma=45 \cdot 10^{-3} \mathrm{~J} \cdot \mathrm{m}^{-2} ; G=48 \mathrm{GPa} ; \boldsymbol{b}=(\sqrt{2} / 2) a$, where $a$ is the lattice parameters; $\boldsymbol{b}_{1}=(\sqrt{6} / 6) a$ [11]. Taking $\alpha=1$, for $\mathrm{n}=1,2$ and $4, D_{\text {c }}$ is estimated to be 34,115 and $277 \mathrm{~nm}$, respectively. These values are in agreement with the experimental observations. Furthermore, it is obvious that the refinement of grain size and the increase of stress concentration benefit the deformation twinning.

On the other hand, for $n=1,2,4, \tau_{\mathrm{p}}$ is estimated to be $0.723,0.215$ and $0.113 \mathrm{GPa}$ at the critical size $D_{\text {c }}$, respectively. During milling, the average pressure introduced by the ball's impact force on the powders can be determined by [12]:

$$
P_{\text {max }}=g_{p} v^{0.4}\left(\rho / E_{\text {eff }}\right)^{0.2} E_{\text {eff }}
$$

where $v$ is the precollision relative velocity $\left(v=6 \mathrm{~m} \cdot \mathrm{s}^{-1}\right.$, in our milling condition), $\mathrm{g}_{\mathrm{p}}$ is a geometrical constant $\left(g_{p}=0.4646\right), \rho$ is the density of balls $\left(\rho_{\mathrm{Fe}}=7.8 \mathrm{~g} \cdot \mathrm{cm}^{-3}\right)$, and $\mathrm{E}_{\text {eff }}$ is the effective modulus of the colliding media $\left(E_{\text {eff-Fe }}=66 \mathrm{GPa}\right)$. Accordingly, we obtain $\mathrm{P}_{\max }=2.57 \mathrm{GPa}$, which is consistent with that of Maurice and Courtney, where $\mathrm{P}_{\max }$ was found to be 2.47-6.18GPa for the various mill configurations $[13,14]$. Since $\mathrm{P}_{\max }$ is larger than $\tau_{\mathrm{p}}$ under the present milling condition, twinning may become the favorable deformation mode. Thus, the theoretical analysis suggests that partial dislocation emission from GBs is a viable deformation mechanism under the present ball milling conditions.

Besides the twinning mechanism of partial dislocation emission from GB, another possible way to nucleate twins has also been observed. Fig. 2 shows another type of twin in the sample milled for 30 h. As shown in Fig. 2 (b), areas I and III, as well as areas II and III form the twin relationship with $\{111\}_{\mathrm{Cu}}$ as the twinning plane, respectively. The GB between areas I and II has been marked by a black line and the orientation between the $\{111\}$ plane of areas I and II is about $74^{\circ}$. This type of twins is formed by the splitting and subsequent migration of a GB segment, leaving behind two twin boundaries. This twinning mechanism is also observed in nanocrystalline Al by MD simulation [15]. It has been suggested that the dissociation of the GB segment into a twin boundary and a new GB is favorable from thermodynamical point, as the energy of twin boundary is smaller than that of GB [16]. More detailed description on the splitting and migration of GBs mechanism can be obtained in refs. $[15,17]$.
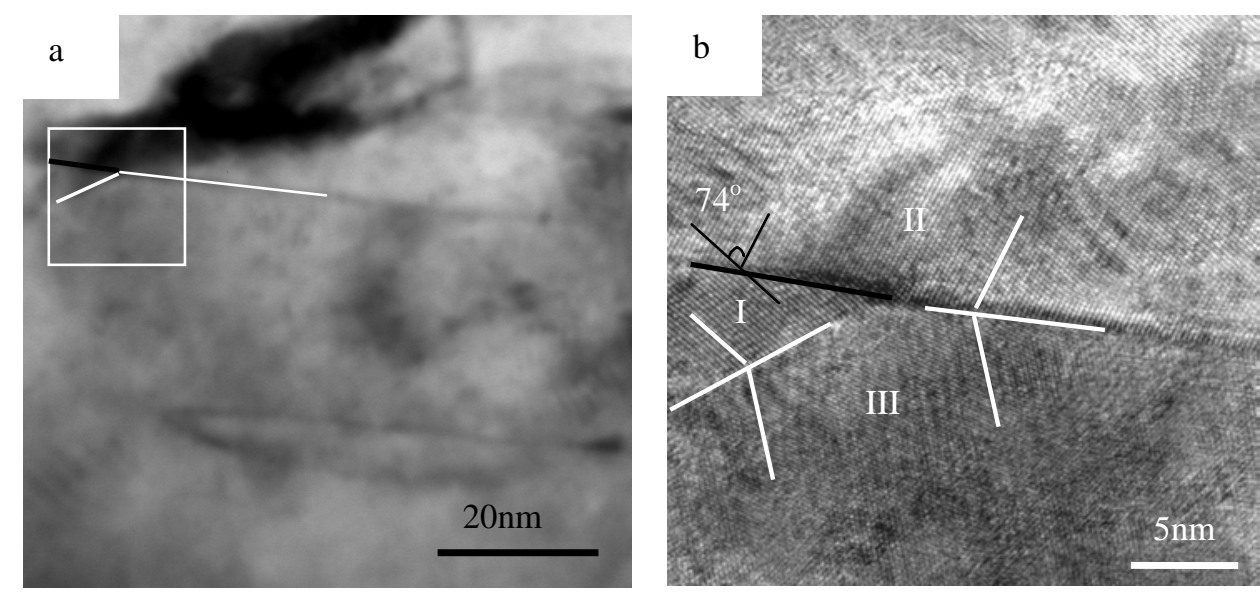

Figure 2. (a) TEM image of the grain containing two twinning relationships; (b) HRTEM image of the region marked by the box in (a). 


\section{Conclusions}

Two types of DTs have been observed in Cu-Nb alloy with grain sizes around or below $100 \mathrm{~nm}$ during ball milling at room temperature. The formation of DTs is found to occur via two different routes: they are nucleated from GBs and grow via successively partial dislocation emission, or the twins form via the dissociation and migration of GB segments. The observed deformation mechanisms demonstrate that the formation of DTs in fcc materials with medium or high SFE depends on the grain size and stress concentration.

\section{Acknowledgments}

This work is supported by the National Natural Science Foundation of China (No. 51401197).

\section{References}

[1] M.J. Szczerba, S. Kopacz, M.S Szczerba, Experimental studies on detwinning of face-centered cubic deformation twins, Acta Mater. 104 (2016) 52-61.

[2] M. W. Chen, E. Ma, J. Kevin, et al., Deformation twinning in nanocrystalline aluminum, Science 300 (2003) 1275-1277.

[3] C.X. Huang, K.Wang, S.D.Wu, et al., Deformation twinning in polycrystalline copper at room temperature and low strain rate, Acta Mater. 54(2006) 655-665.

[4] X.Z. Liao, F. Zhou, E.J. Lavernia, et al., Deformation twins in nanocrystalline Al, Applied Physics Letters 83 (2003) 5062-5064.

[5] M.D. Abad, S. Parker, D. Kiener, et al., Microstructure and mechanical properties of $\mathrm{Cu}_{\mathrm{x}} \mathrm{Nb}_{1-\mathrm{x}}$ alloys prepared by ball milling and high pressure torsion compacting, Journal of Alloys and Compounds 630 (2015) 117-125.

[6] E. Botcharova, J. Freudenberger, L. Schultz, Mechanical and electrical properties of mechanically alloyed nanocrystalline Cu-Nb alloys, Acta Mater. 54 (2006) 3333-3341.

[7] S. Mula, H. Bahmanpour, S. Mal, et a.l, Thermodynamic feasibility of solid solubility extension of $\mathrm{Nb}$ in $\mathrm{Cu}$ and their thermal stability, Materials Science and Engineering A 539 (2012) 330-336.

[8] R.S. Lei, M.P. Wang, Z. Li, et al., Structure evolution and solid solubility extension of copper-niobium powders during mechanical alloying, Materials Science and Engineering A 528 (2011) 4475-4481.

[9] J. P. Hirth, J. Lothe, Theory of dislocations, Wiley, New York, 1982.

[10] L. Lu, X. Chen, X. Huang, K. Lu, Revealing the maximum strength in nano-twinned copper, Science 323 (2009) 607-610.

[11]J.Y. Huang, Y.K. Wu, H.Q. Ye, Deformation structures in ball milled copper, Acta mater. 44 (1996) 1211-1221.

[12]D.R. Maurice, T.H. Courtney, The physics of mechanical alloying: A first report, Metall. Trans. A 121 (1990) 289-303.

[13] E. H. Love, A Treatise on the Mathematical Theory of Elasticity, Dover, New York ,1994.

[14] S.P. Timoshenko, J.N. Goodier,Theory of Elasticity , McGraw-Hill, New York, 1970.

[15] V. Yamakov, D. Wolf, R. S. Phillpot, Deformation twinning in nanocrystalline Al by molecular dynamics simulation, Acta Mater. 50 (2002) 5005-5020.

[16] S. Mahajan, C.S. Pande, M.A. Imam, B.B. Rath, Formation of annealing twins in f.c.c. crystals, Acta mater. 45 (1997) 2633-2638.

[17]V. Yamakov, D. Wolf, R. S. Phillpot, Dislocation processes in the deformation of nanocrystalline aluminium by molecular-dynamics simulation, Nature Materials 1 (2002) 1-4. 